\title{
Entrelacs
}

Cinéma et audiovisuel

18 | 2021

Laurent Roth, cinéaste

\section{Laurent Roth, Petit Prince à la caméra}

\section{Marc-Antoine Vaugeois}

\section{OpenEdition}

Journals

Édition électronique

URL : https://journals.openedition.org/entrelacs/6177

DOI : 10.4000/entrelacs.6177

ISSN : 2261-5482

Éditeur

Éditions Téraèdre

Référence électronique

Marc-Antoine Vaugeois, « Laurent Roth, Petit Prince à la caméra », Entrelacs [En ligne], 18| 2021, mis en ligne le 14 décembre 2021, consulté le 14 décembre 2021. URL : http://journals.openedition.org/ entrelacs/6177 ; DOI : https://doi.org/10.4000/entrelacs.6177

Ce document a été généré automatiquement le 14 décembre 2021.

Tous droits réservés 


\title{
Laurent Roth, Petit Prince à la caméra
}

\author{
Marc-Antoine Vaugeois
}

« S'il vous plaît... dessine-moi un mouton! » La demande, exprimée sans préambules par le Petit Prince à Saint-Exupéry, pourrait représenter le geste de cinéaste de Laurent Roth, qui n'aime rien moins que décliner cette situation dans la plupart de ses films. Comme il n'est pas satisfait, l'enfant transforme sa demande en jeu, en poussant l'aviateur perdu au milieu du désert à s'y reprendre plusieurs fois pour produire une image, et à faire preuve d'imagination pour trouver la solution - il lui faut dessiner autre chose qu'un mouton... On retrouve cette même nécessité de transformer la question posée en jeu chez Laurent Roth, afin que les participants réunis à l'intérieur de ses films se rencontrent et se mettent au travail, tous ensemble. S'élabore alors un cinéma qui se fait " côte à côte ", qui est rendu possible parce que deux corps (ou plus) sont mis en présence et installés l'un à côté de l'autre. C'est la " genèse » de tous les films, qui renvoie au couple originel des récits bibliques, inlassablement réinventé par le réalisateur. Sauf qu'ici, c'est à Adam et Eve qu'il reviendrait de dessiner leur jardin d'Éden. 


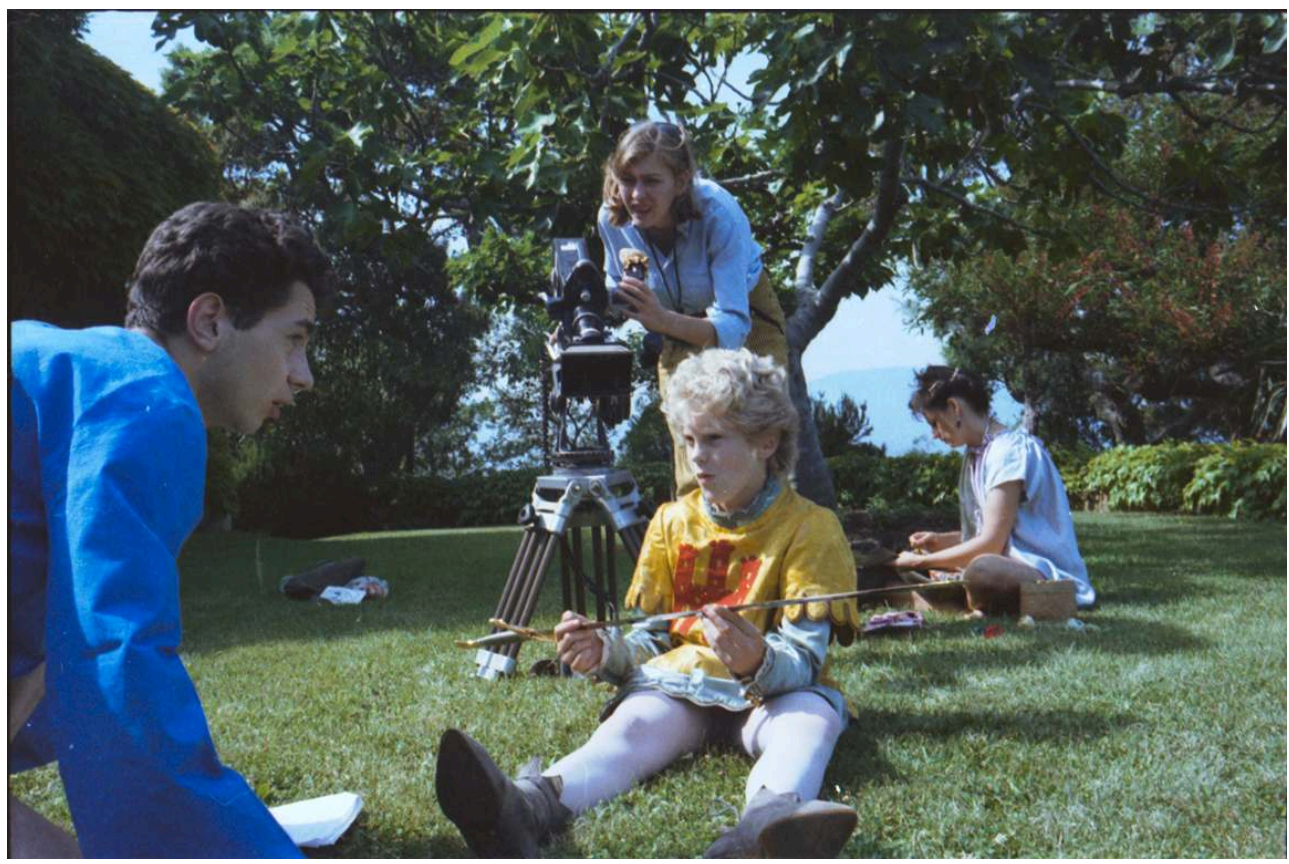

Tournage de Marie ou le retour (1984).

Qu'il réunisse ses proches pour une enquête sur ses racines familiales, qu'il monte une troupe de comédiens pour plonger avec eux dans les écrits du metteur en scène de théatre Jacques Copeau et retracer le récit de sa vie aventureuse, ou qu'il invite le cinéaste Amos Gitaï à s'entretenir avec lui à propos de son impressionnante carrière, Laurent Roth élabore une série de protocoles singuliers pour faire advenir et mettre en scène la parole, la sienne et celle des autres. Cela relève presque toujours de ce que l'on appelle communément un "travail à la table », bien qu'il arrive souvent que soit fait l'économie d'une table et que l'on se contente de s'asseoir sur un banc ou sur des chaises, à l'air libre ou en intérieur. Sitôt que l'on s'assied, on peut se mettre à l'ouvrage, dérouler le fil de la pensée et des souvenirs dans une discussion à bâtons rompus ou au travers de la lecture, en s'appuyant sur différents supports qui relancent et nourrissent le dialogue: textes, photographies ou accessoires divers, inattendus parfois, comme les jouets de construction que le cinéaste étale devant chaque membre du clan Roth, invité à reconstituer à tour de rôle, de mémoire et sous l'œil avide du fils prodigue, une image de leur maison de famille au moyen de ces jouets d'enfants. À cet instant ne compte plus que la disposition dans l'espace des corps, qu'il s'agit d'agencer les uns par rapport aux autres pour en diriger l'expression. 


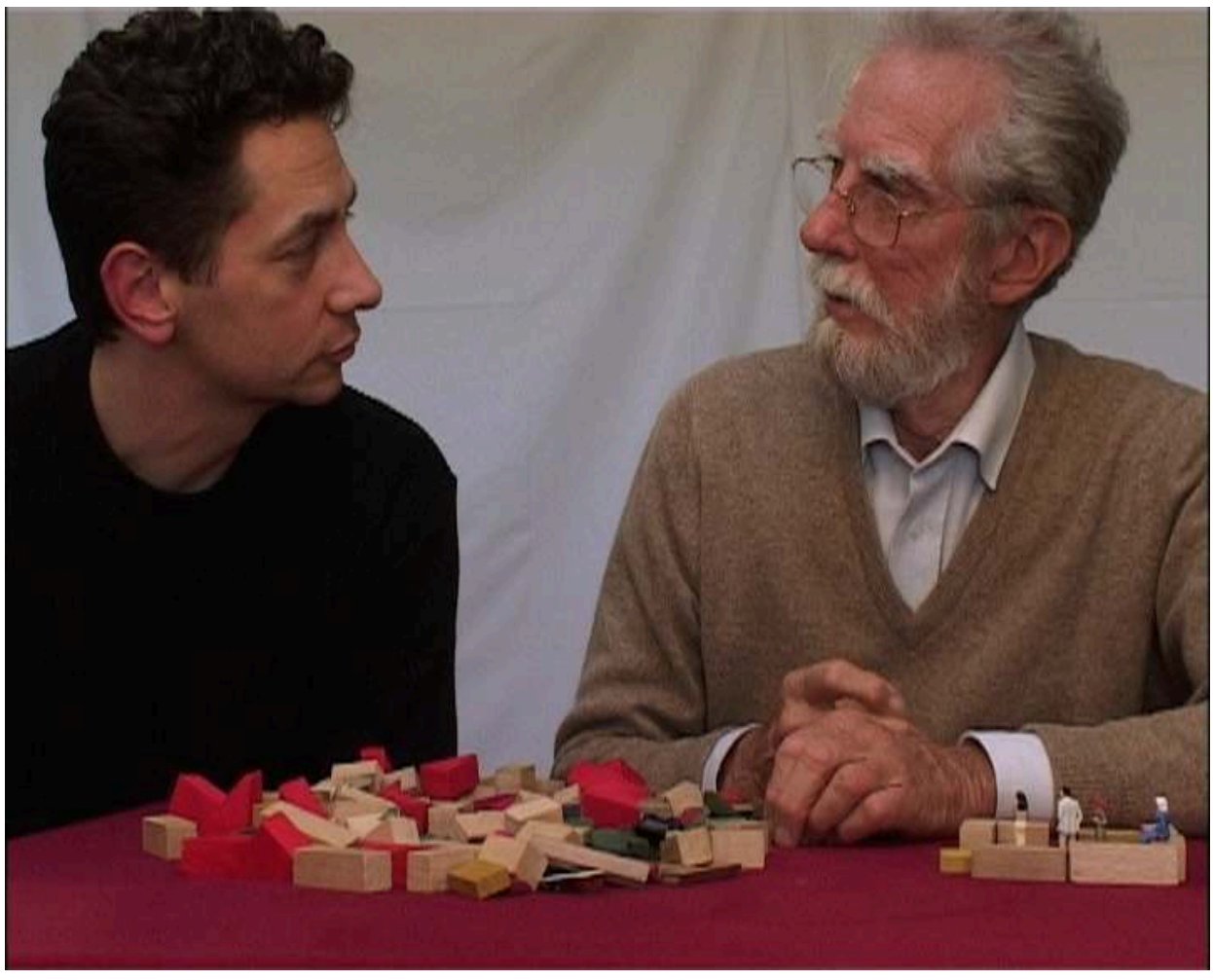

Une maison de famille (2004).

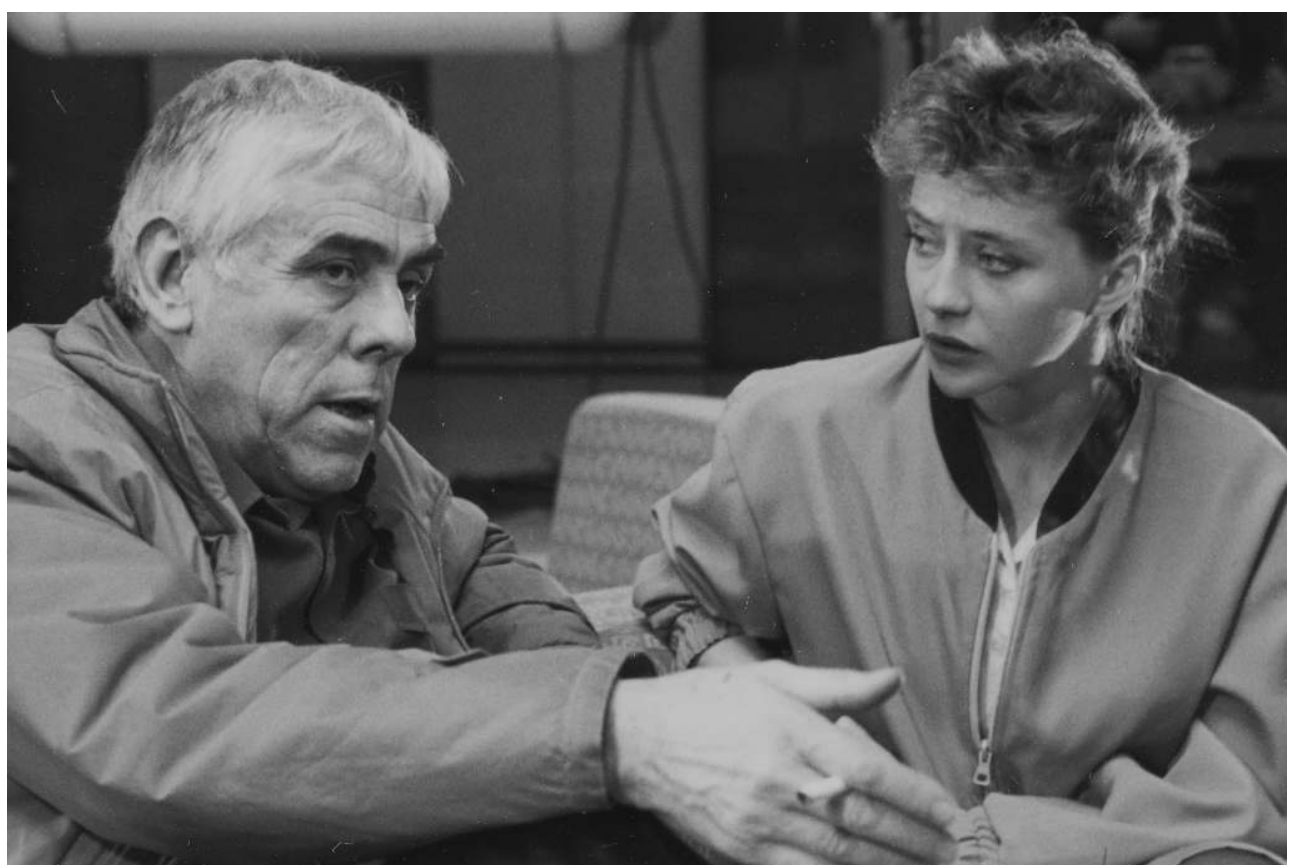

Raoul Coutard et Mireille Perrier dans Les Yeux brûlés (1986).

3 Les participants des films de Laurent Roth se révèlent à chaque fois moins déstabilisés par les règles des jeux qu'on leur propose que par cette demande qui leur est faite d'accepter d'être vus en train de jouer, en présence d'un partenaire qui les accompagne, les bouscule ou les encourage dans cette entreprise. Cette donnée a pour principal effet d'oblitérer tout rapport de déférence attendu vis-à-vis des figures convoquées, qui font souvent autorité dans leur domaine au regard d'un savoir ou 
d'une expérience dont ils sont les dépositaires. Il en va des grands maîtres interrogés sur leur pratique artistique et journalistique (Amos Gitaï, Henri Alekan, les reporters de guerre des Yeux brûlés) comme de l'entourage familial du cinéaste. Il leur faut alors composer leurs réponses avec d'autres éléments visibles, avec les moues et la fébrilité frondeuse de Mireille Perrier pour les photographes baroudeurs, contraints de baisser la garde face à la jeune femme qui les interroge sur leurs faits d'armes journalistiques, ou encore avec la voix douce du metteur en scène lui-même, opposant par sa présence dans son récent entretien avec le mogul Amos Gitaï, un contrepoint nécessaire pour que la parole du cinéaste israélien ne croule pas sous sa propre superbe - on serait tenté de parler également de contrepoids, tant les deux hommes se valent en terme de carrure, et que l'équilibre des plans semble reposer là-dessus, sur les positions de ces deux corps imposants légèrement inclinés l'un vers l'autre, ne laissant naviguer que leurs regards depuis l'écran où défilent des extraits de films et des archives personnelles de Gitaï.

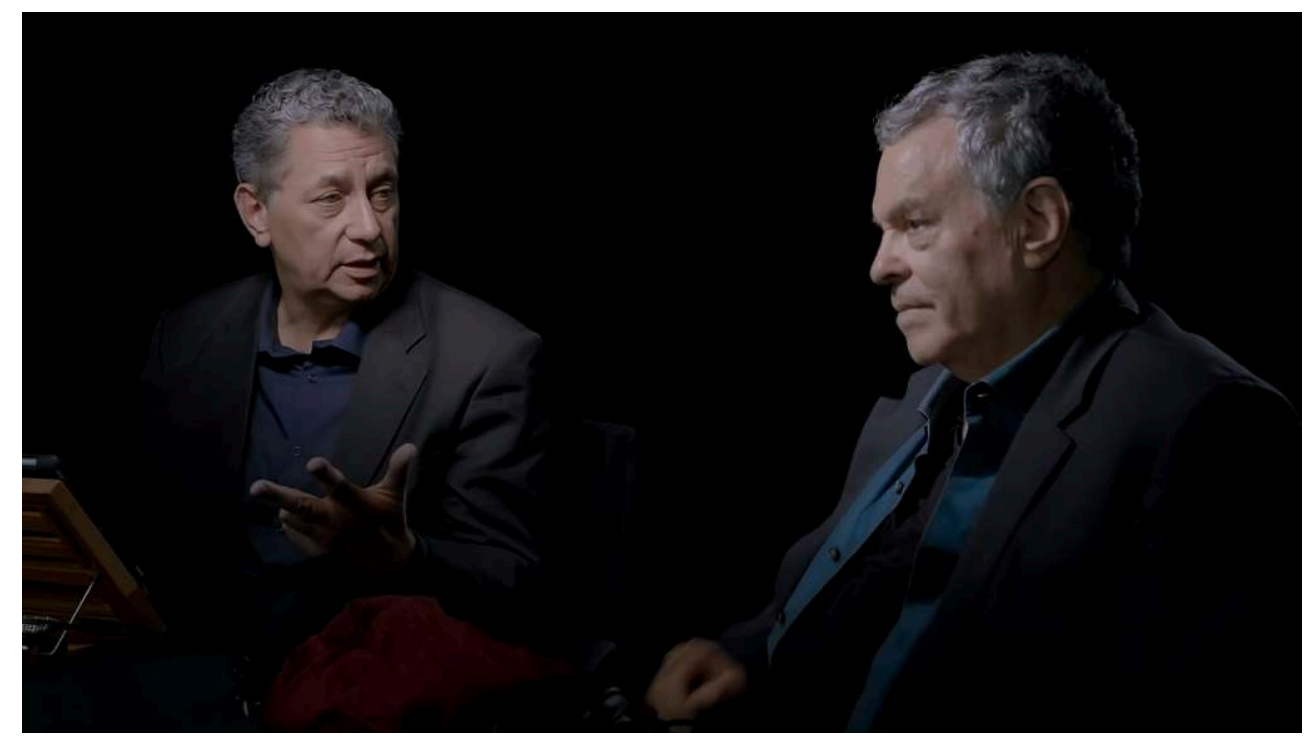

Amos Gitaï, la violence et I'histoire (2020).

$4 \quad$ S'il est résolument plus approprié de parler de jeux que de dispositifs à propos du travail de Laurent Roth, c'est parce que ce dernier se garde bien de toute tentation démiurgique, s'amusant même ouvertement de cette position - comme dans L'Impromptu de Jacques Copeau (1993) où la silhouette fantasmatique du metteur en scène se découpe dans la pénombre, toisant depuis sa régie la scène de théatre et la petite troupe de comédiens qu'il dirige, produisant un effet de surplomb trop appuyé pour que l'on n'y décèle pas une certaine ironie. Il faut bel et bien en passer par-là, par cette mise en berne de la toute-puissance du metteur en scène, et de toute figure d'autorité en général, pour qu'une force organisatrice s'exerce de façon diffuse, permettant à chacun des acteurs du film de se déprendre un moment de lui-même, en faisant de la place à celui qui se tient près de lui ou qui regarde par-dessus son épaule, acceptant d'en faire son camarade de jeu pour enfin dessiner le mouton, à deux. 


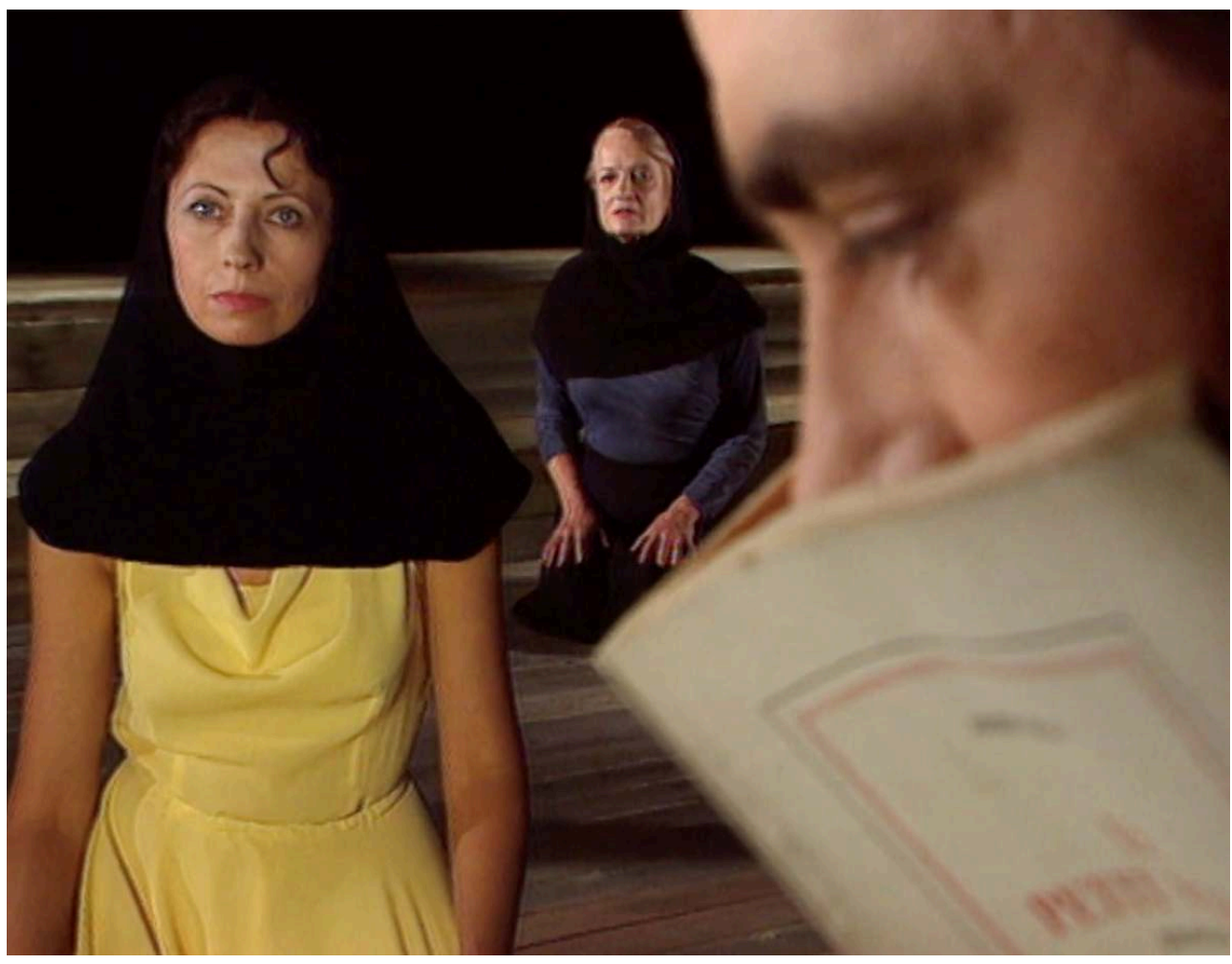

L'Impromptu de Jacques Copeau (1993). Laurent Roth, Marie Rivière et Catherine Dasté dans un jeu théâtral filmé : Le Petit Pauvre.

Crédit images : Laurent Roth.

\section{RÉSUMÉS}

Cet article propose une réflexion sur l'œuvre de Laurent Roth, en définissant la méthodologie employée par le cinéaste dans chacun de ses films et sa capacité à la renouveler. Nous verrons quelle importance y revêt la notion de «jeu», comme condition de la mise au travail des différents partis et comme moyen ludique et singulier de mener l'enquête au sein d'une écriture documentaire.

This article proposes a reflection on the work of Laurent Roth, defining the methodology employed by the filmmaker in each of his films and his capacity to renew it. We will see how important the notion of "play" is, as a condition for putting the different parties to work and as a playful and singular means of conducting the investigation within a documentary script.

\section{AUTEUR}

\section{MARC-ANTOINE VAUGEOIS}

Réalisateur, acteur et critique de cinéma. Il a joué dans de nombreux courts et longs-métrages, sous la direction notamment de Justine Triet, de Lucie Borleteau, de Pascale Bodet ou encore 
d'Ilan Klipper. De 2013 à 2017, il rédige des textes critiques et réalise des entretiens avec des cinéastes pour différentes revues (Format Court, À bras le corps, Répliques). Il est également l'auteur de trois courts-métrages. 